\title{
On the Value of Measuring Dopamine, Norepinephrine and Their Metabolites in Schizophrenia
}

René S. Kahn, M.D. and Michael Davidson, M.D.

The two papers by Maas et al. $(1993 a, b)$ highlight both the valuable information that can be obtained by measuring catecholamines and their metabolites in cerebrospinal fluid (CSF), plasma, and urine, and the conceptual and practical limitations of using peripheral measurements to reflect central neuronal function.

The effort to find peripheral measures that reflect central monoamine (MA), and particularly dopamine (DA) function, has been motivated by the DA hypothesis of schizophrenia, which in turn originated from data on the affınity of neuroleptics to DA receptors (Carlsson and Lindquist 1963) and the effect of neuroleptics on DA turnover (Bunney and Grace 1978). However, the notion that catecholamine concentrations in remote body fluids are indicative of central neuronal activity is based on a cascade of assumptions, such as, changes in MA turnover reflect neurotransmission and intracellular processes; changes in turnover at the level of the synaptic cleft are not offset by rapid adaptive mechanisms; and the size of change in MA turnover in discrete

large compartments like CSF, plasma, or urine. Moreover, the idea that in patients grouped around ambiguous clinical similarities (like most psychiatric disorders) detection of an isolated catecholamine abnormality during a single measurement (as in most studies) can reveal pathophysiologic abnormalities is equally problematic.

\footnotetext{
From the Bronx Veterans Affairs Medical Center, and Mount Sinai Medical School, New York, New York.

Address correspondence to: Michael Davidson, M.D., Clinical Research Division, Bronx Veterans Affairs Medical Center, Psychiatry Service 116A, 130 West Kingsbridge Road, Bronx, New York 10468. Received September 18, 1992; accepted September 21, 1992.
}

Nevertheless, some highly consistent findings have been produced when DA metabolites were measured in CSF and plasma prior to and during neuroleptic treatment. For instance, seven of eight CSF studies have found neuroleptic treatment to increase homovanillic acid (HVA) and all three studies that examined this issue have found this increase to be unrelated to treatment response (see Kahn et al. 1992). Similarly, in measuring HVA in plasma all seven studies found neuroleptic treatment to lower plasma-HVA concentrations and all found this decrement to relate to treatment outcome (Davis et al. 1991).

In contrast, studies examining HVA during the steady state have produced less consistent results. Cerebrospinal fluid HVA has generally not been found to differentiate schizophrenic patients from healthy control subjects, nor has it been found to relate to specific schizophrenic symptoms or subgroups (Widerlov 1988). Similarly, plasma-HVA concentrations differentiate patients from control subjects only in some studies, and the results of studies that tried to link plasma-HVA concentrations to specific schizophrenic symptoms, or even to severity of illness, have also been contradictory (see Davis et al. 1991).

Thus, although the results are conflicting when HVA is used as an indication of baseline DA furiction, when HVA is used as an index of change in DA function, results are remarkably uniform. The latter consistency may hinge on the relatively large changes in HVA production when DA activity is manipulated. For instance, administration of $1 \mathrm{mg} / \mathrm{kg}$ of apomorphine in rats reduces striatal HVA by about one-third, and by about $25 \%$ in plasma (Kendler et al. 1982). Haloperidol $(1 \mathrm{mg} / \mathrm{kg}$ ) almost quadruples HVA in the striatum and nearly doubles it in the plasma of rats (Kendler et al. 
1981). A single administration of haloperidol roughly doubles plasma-HVA concentrations in human subjects (Davila et al. 1987). Thus, the changes induced by perturbation of DA function lead to large changes in both central and peripheral HVA concentrations. Possibly, when DA function is manipulated, the changes that occur are profound enough to be detected in metabolite concentrations in CSF or plasma. In contrast, when steady-state DA function is assessed, DA metabolite concentrations may be much more prone to multiple confounding factors. These are indeed legion. Cerebrospinal fluid metabolite concentrations are affected by age, height, and weight (Hartikainen et al. 1991) as well as by circadian (Nicoletti et al. 1981) and seasonal (Losonczy et al. 1984) variations. As explained in the papers by Maas et al. (1993a,b) only $15 \%$ to $35 \%$ of plasma HVA may be derived from central sources, and consequently plasma HVA may reflect central DA turnover only when central DA metabolism is drastically altered.

Interestingly, measurement of HVA in plasma does not replace measurement of HVA in CSF or vice versa. Indeed, the studies examining the effect of treatment on HVA have provided quite different, if not opposite, results depending upon whether HVA was measured in CSF or plasma: CSF HVA increases with neuroleptic treatment whereas plasma HVA decreases; the increase in CSF HVA is unrelated to treatment outcome, but the decrease in plasma HVA consistently correlates with symptomatic improvement. These consistent, although opposite findings may be meaningful and should stimulate further studies. For instance, does it reflect differential effects of neuroleptics on various brain areas? Presently, it is unknown from which brain area CSF HVA is primarily derived. Indeed, even the basic question of whether MA (metabolites) measured in lumbar CSF reflect brain CSF concentrations is unclear. Although two human post-mortem studies found MA metabolite concentrations in ventricular CSF to correlate with those in brain tissue (Stanley et al. 1985; Wester et al. 1990), correlations between cisternal and lumbar MA metabolite concentrations have only been found in some (Degrell and Nagy 1990), but not other studies (Maraia et al. 1975). (Interpretation of these studies is hampered, however, because they examined [neurologically] ill, not healthy subjects). It has been proposed that due to the large area, CSF HVA is of cortical rather than subcortical origin. This assumption appears to be supported by the finding that in schizophrenic patients, blood flow in the dorsolateral prefrontal cortex was correlated with CSF HVA (Weinberger et al. 1988). In monkeys, CSF HVA reflects HVA in frontal cortical but not other cortical areas (Elsworth et al. 1987). What, if any, brain area is reflected in plasmaHVA concentrations in man is unstudied, although it has been proposed that plasma HVA reflects subcorti- cal DA activity rather than cortical DA activity (Pickar et al. 1992). Measurement of HVA in CSF and plasma may provide distinct windows to separate brain areas. That this may be so, is suggested by a recent study that found a correlation between CSF HVA and negative symptoms (hypothesized to be related to frontal cortical dopaminergic deficits) whereas positive symptoms (hypothesized to be associated with increased subcortical DA activity) were correlated with plasma-HVA concentrations (Pickar et al. 1992).

Simultaneous assessment of CSF- and plasmaHVA ccencentrations, possibly in combination with cerebral blood flow measurements, prior to and during (atypical) neuroleptic treatment may also contribute to elucidating the mechanism(s) of action of typical and atypical antipsychotics. For instance, do neuroleptics increase DA turnover in mesocortical DA neurons (expressed as increased CSF HVA) and decrease it in mesolimbic DA neurons (expressed as decreased plasma HVA)? If so, is the increase in CSF HVA related to reduction in negative symptoms and/or with im. provement in cognitive performance? Conversely, is the decrease in plasma HVA associated with a reduction in positive symptoms? And finally, do atypical neuroleptics display opposite effects on CSF and plasma HVA as well?

In summary, measurement of MA metabolite concentrations in CSF and plasma has produced consistent results when examined in relationship to neuroleptic treatment. Although measurement of MA metabolites in CSF and plasma is fraught with theoretical limita. tions, in practice it proves to be, at the very least, a promising tool to help elucidate the mechanism of action of (atypical) neuroleptics.

\section{REFERENCES}

Bunney BS, Grace AA (1978): Acute and chronic haloperidol treatment: Comparison effects on nigral dopaminergic cell activity. Life Sci 23:1715-1728

Carlsson A, Lindquist M (1963): Effect of chlorpromazineand haloperidol on formation of 3-methoxytyramine and normetanephrine in mouse brain. Acta Pharmacol Toxicol 20:140-144

Davila R, Zumarraga M, Perea K, Andia I (1987): Elevation of plasma homovanillic acid level can be detected within four hours after initiation of haloperidol treatment. Arch Gen Psychiatry 44:837-838

Davis KL, Kahn RS, Ko G, Davidson M (1991): Dopamine and schizophrenia: A review and reformulation. Am ) Psychiatry 148:1474-1486

Degrell I, Nagy E (1990): Correlations between cisternal CSF and plasma concentrations of HVA, MHPG, 5HIAA, DA and NA. Biol Psychiatry 27:1179-1182

Elsworth JD, Leahy DJ, Roth RH, Redmond DE Jr (1987): Homovanillic acid concentrations in brain, CSF and 
plasma as indicators of central DA function in primates. J Neural Transm 68:51-62

Hartikainen P, Soininen H, Reinikainen KJ, SirvioJ, Soikkeli R, Riekkinen PJ (1991): Neurotransmitter markers in the cerebrospinal fluid of normal subjects: Effects of aging and other confounding factors. J Neural Transm 84: 103-117

Kahn RS, Davidson M, Knott P, Stern RG, Apter SA, Davis KL (1992): Effect of neuroleptic medication in cerebrospinal fluid monoamine metabolite concentrations in schizophrenia: Serotonin-dopamine interactions as a target for treatment. Arch Gen Psychiatry (in press)

Kendler KS, Heninger GR, Roth RH (1981): Brain contribution to the haloperidol-induced increase in plasma homovanillic acid. Eur J Pharmacol 71:321-326

Kendler KS, Heninger GR, Roth RH(1982): Influence of dopamine agonists on plasma and brain levels of homovanillic acid. Life Sci 30:2063-2069

Losonczy MF, Mohs RC, Davis KL (1984): Seasonal variations of human lumbar CSF neurotransmitter metabolite concentrations. Psychiatry Res 12:79-87

Maas JW, Contreras SA, Miller AI, Berman N, Bowden CL, Javors MA, Seleshi E, Weintraub S (1993a): Studies of catecholamine metabolism in schizophrenic/psychosis I. Neuropsychopharmacology, 8:97-109

Maas JW, Contreras SA, Miller AI, Berman N, Bowden CL, Javors MA, Seleshi E, Weintraub S (1993b): Studies of catecholamine metabolism in schizophrenic/psychosis II. Neuropsychopharmacology, 8:111-116
Maraia G, Bureggi SR, di Rocco C, Calderini G, Morselli PL (1975): Monoamine acid metabolites and cerebrospinal fluid dynamics in normal pressure hydrocephalus: Preliminary results. J Neurol Neurosurg Psychiatry 38:123-128

Nicoletti F, Raffaele R, Falsaperla A, Paci R (1981): Circadian variation in 5HIAA levels in human cerebrospinal fluid. Eur Neurol 20:9-12

Pickar D, Owen RR, Litman RE, Konicki PE, Gutierrez R, Rapaport $\mathrm{MH}$ (1992): Clinical and biological response to clozapine in patients with schizophrenia: Crossover comparison with fluphenazine. Arch Gen Psychiatry 49: 345-353

Stanley M, Traskman-Bendz L, Dorovini-Zis K(1985): Correlations between aminergic metabolites simultaneously obtained from human CSF and brain. Life Sci 37: 1279-1286

Weinberger DR, Berman KF, Illowsky BP (1988): Physiological dysfunction of dorsolateral prefrontal cortex in schizophrenia. III. A new cohort and evidence for a monoaminergic mechanism. Arch Gen Psychiatry 45:609-615

Wester P, Bergstrom U, Eriksson A, Gezelius C, Hardy J, Winblad B (1990): Ventricular cerebrospinal fluid monoamine transmitter and metabolite concentrations reflect human brain neurochemistry in autopsy cases. J Neurochem 54:1148-1156

Widerlov E (1988): A critical appraisal of CSF monoamine metabolite studies in schizophrenia. Ann NY Acad Sci 537:309-323 